\title{
Distinctive profiles of tumor-infiltrating immune cells and association with intensity of infiltration in colorectal cancer
}

\author{
YUGANG WU, LEI YUAN, QICHENG LU, HAIYAN XU and XIAOZHOU HE \\ Department of Surgery, The Third Affiliated Hospital of Soochow University/The First \\ People's Hospital of Changzhou, Changzhou, Jiangsu 213000, P.R. China
}

Received May 28, 2016; Accepted July 27, 2017

DOI: $10.3892 / \mathrm{ol} .2018 .7771$

\begin{abstract}
Tumor-infiltrating immune cells are heterogeneous and consist of characteristic compartments, including $\mathrm{T}$ helper (Th) 1 and regulatory $\mathrm{T}$ (Treg) cells that exhibit distinctive biological functions. The present study investigated the profile of infiltrating immune cells from surgically removed tumor tissues from patients with colorectal cancer. The characteristic transcription factors of Th1 and Th2 cells, Treg cells, Th17 cells and $\mathrm{T}$ follicular helper (Tfh) cells were analyzed. The results demonstrated that a marked increased number of Treg cells presented in tumor infiltrates when compared with non-tumor adjacent tissues. An increased number of Th1 and Tfh cells existed in tumor infiltrates compared with non-tumorous adjacent tissues, while the infiltration of Th17 and Th2 cells was similar between tumor and non-tumor adjacent tissues. Furthermore, there were an increased number of Treg cells in tumors with low infiltration compared with those with high infiltration. The expression of CXC motif chemokine (CXC) receptor 3, CXC ligand (CXCL)L9 and CXCL10 was significantly increased on infiltrating $\mathrm{T}$ cells in tumors with high infiltration as compared with those with low infiltration. Macrophages exhibited a dominant M2 phenotype in tumor infiltrates of colorectal cancer, whereas a balanced M1 and M2 phenotype presented in macrophages from the peripheral blood. In vitro stimulation of macrophages isolated from tumor tissue of colorectal cancer with granulocyte macrophage colony-stimulating factor and lipopolysaccharide did not drive to an inflammatory phenotype. The results provide insights into the pattern of immune cell infiltration in Chinese patients with colorectal cancer. It may be beneficial that patients with colorectal cancer are screened for the defined profile along with the expression of CXCL9 and CXCL10 in order to achieve better efficacy in clinical applications of
\end{abstract}

Correspondence to: Dr Xiaozhou He, Department of Surgery, The First People's Hospital of Changzhou, 185 Juqian Street, Changzhou, Jiangsu 213000, P.R. China

E-mail: wuyugang89@163.com

Key words: colorectal cancer, macrophage, T cell, infiltration immune-based therapy, including anti-programmed cell death protein 1 therapy.

\section{Introduction}

Colorectal cancer (CRC) is a common malignant disease, which has been intensely studied for tumor-immune interactions in order to develop successful immunotherapies. In particular, systemic $\mathrm{T}$ cell responses against tumor antigens and tumor-infiltrating $\mathrm{T}$ cells have been analyzed in detail in CRC (1-4). A number of studies have linked a high $\mathrm{T}$ cell infiltration to an improved survival in CRC (1-6). Patients with $\mathrm{CRC}$ as well as those with other malignant diseases are able to mount an antigen-specific $\mathrm{T}$ cell response without prior immunotherapy $(7,8)$. Peripheral tumor-associated antigen-directed $\mathrm{T}$ cell responses were observed to have no survival benefit for patients with colorectal cancer despite of a limited number of patients studied (9). Various components, including the immune system, tumor stroma and tumor cells affect the induction and modulation of tumor-directed immune responses (10). Limited antitumor activity of spontaneous antigen-specific $\mathrm{T}$ cells at a clinical level in patients with CRC may be due to multiple factors. Investigating the profiles of infiltrating immune cells may help to understand the interaction between innate and adaptive immune response and improve immunotherapeutic approaches in CRC.

Traditionally, cluster of differentiation (CD) $8^{+}$cytotoxic T cells have been considered as the key component of effective antitumor immunity, and breast tumors with higher levels of infiltrating $\mathrm{CD}^{+} \mathrm{T}$ cells have been associated with improved patient survival $(11,12)$. However, studies have also shown that $\mathrm{CD}^{+} \mathrm{T}$ cells frequently fail to fully function in vivo if there is a lack of adequate assistance from $\mathrm{CD} 4^{+} \mathrm{T}$ cells (13). Therefore, heterogeneous populations of infiltrating immune cells need to be clarified in order to understand the antitumor immune responses within tumor.

The current consensus is that interferon (IFN)- $\gamma$-producing $\mathrm{CD}^{+} \mathrm{T}$ helper $(\mathrm{Th}) 1$ and $\mathrm{CD} 8^{+} \mathrm{T}$ cells, along with mature dendritic cells (DCs), natural killer (NK) cells, M1 macrophages and type $1 \mathrm{NK}$ T cells are able to generate effective but frequently attenuated anti-tumor responses, while $\mathrm{CD}^{+}$ Th2 cells and type 2 NK T cells in cooperation with $\mathrm{CD}^{+}$ Tregs (regulatory), myeloid-derived suppressor cells, immature DCs or M2 macrophages suppress antitumor immunity 
and are able to promote tumor progression (14-16). However, this summarized observation comes with the caveat that variation exists among tumor types, with the pro-tumorigenic cells, including CD4 ${ }^{+}$Th17, also shown to produce effective antitumor responses $(17,18)$.

The present study was undertaken to characterize the immune cell subpopulations infiltrating human breast tumors in a direct ex vivo analysis of fresh tumor tissue short-term in vitro expansion. In the present study, a profile of tumor-infiltrating T cells and macrophages in human CRC was analyzed. A broad spectrum of markers was applied to distinguish two subsets of macrophages. In addition, it was examined whether tumor macrophages were prone to cytokine-driven conversion. In addition, the expression of CXC motif chemokine (CXC) receptor 3 (CXCR3), CXC ligand (CXCL) 9 and CXCL10 was analyzed. These important molecules were associated with the intensity of infiltration. The results provided insights into the profile of infiltrating immune cells in human CRC and may be useful for further study of antitumor immune responses in human CRC.

\section{Materials and methods}

Patients and specimens. Subsequent to approval from the institutional review board of the First People's Hospital of Changzhou (Changzhou, China) and informed consent, surgically removed tissue blocks and peripheral blood mononuclear cells were collected from patients with colorectal cancer from the aforementioned hospital $(n=22,12$ females and 10 males; age range, 52-79 years; median age 63 years; samples collected between April 2015 and March 2016). All analyses were performed in compliance with the Declaration of Helsinki. The demographic information of patients is described in Table I.

Isolation of infiltrating immune cells. Fresh tumor and non-tumorous tissue adjacent were harvested in sterile condition from patients during surgery and rinsed with cold PBS to remove blood clogs, fat tissue and surrounding necrotic tissue. The tissues were then dried with filter papers and weighed. The tissues were cut into small pieces (size, $\sim 1 \mathrm{~mm}^{3}$ ) in cold PBS. In total, $\geq 5$ volumes of collagen IV $(0.1 \mu \mathrm{g} / \mathrm{ml}$ in RPMI-1640) was added to 1 volume of tissue suspension and then incubated at $4^{\circ} \mathrm{C}$ overnight. The tissue suspension was filtered through a nylon mesh (70-100 $\mu \mathrm{m})$ to harvest single cells. Subsequent to washing with PBS, the mononuclear cells were isolated by gradient centrifugation with Percoll ${ }^{\circledR}$ Plus (GE Healthcare Life Sciences, Little Chalfont, UK) at $400 \mathrm{x}$ g at room temperature for $25 \mathrm{~min}$ and counted with an Axiovert 100 inverted microscope (Carl Zeiss AG, Oberkochen, Germany) at x10 magnification. The results were expressed using a heat map for the intensity of infiltration with HemI software (HemI Illustrator; version 1.0.3.3; hemi.biocuckoo.org).

Isolation of macrophages and T cells. Mononuclear cells were suspended in $\mathrm{pH} 7.4 \mathrm{PBS}$ at a density of $>5 \times 10^{5}$ cells $/ \mathrm{ml}$ and then incubated with anti-CD14 microbeads (Miltenyi Biotec GmbH, Bergisch Gladbach, Germany; Cat\#130-050-201) for $30 \mathrm{~min}$ at room temperature. Subsequent to washing, the resuspended cells passed through the MS cell separation column to separate macrophages and other cells according
Table I. Demographics of surgical patients with colorectal cancer.

\begin{tabular}{lcc}
\hline & \multicolumn{2}{c}{ Degree of infiltration } \\
\cline { 2 - 3 } Parameters & $\begin{array}{c}\text { With LN } \\
\text { infiltration }\end{array}$ & $\begin{array}{c}\text { No LN } \\
\text { infiltration }\end{array}$ \\
\hline Total, n & 7 & 15 \\
Sex, $\mathrm{n}$ & & \\
Male & 2 & 8 \\
Female & 5 & 7 \\
Mean age, years & 62.4 & 64.7 \\
Location of tumor, $\mathrm{n}$ & & 5 \\
Ascending colon & 0 & 3 \\
Descending colon & 0 & 0 \\
Transverse colon & 1 & 2 \\
Sigmoid colon & 2 & 5 \\
Rectum & 3 & \\
\hline
\end{tabular}

LN, lymph node.

to the manufacturer's protocol. For T cell isolation, the cells were incubated with anti-CD3 microbeads at $4^{\circ} \mathrm{C}$ for $30 \mathrm{~min}$ (Miltenyi Biotec GmbH; Cat\# 130-050-101) prior to following the procedure as aforementioned.

Reverse transcription-quantitative polymerase chain reaction $(R T-q P C R)$. Total RNA was extracted from cells with an RNeasy Mini kit (Qiagen, Inc., Valencia, CA, USA) according to the manufacturer's instruction. cDNA was then synthesized with the iScript cDNA Synthesis RT kit (Bio-Rad Laboratories, Inc., Hercules, CA, USA), according to the manufacturer's protocol. The specific primers were designed and purchased from Sangon Biotech Co., Ltd. (Shanghai, China). Gene expression profile was analyzed by RT-qPCR with customized primer sets as described in Table II. Briefly, PCR was performed using $10 \mathrm{ng}$ cDNA, $500 \mathrm{nM}$ forward and reverse primers, and SYBR Green master mix (Applied Biosystems; Thermo Fisher Scientific, Inc., Waltham, MA, USA) in $20 \mathrm{ml}$ reactions. Thermocycling conditions comprised an initial holding at $50^{\circ} \mathrm{C}$ for $2 \mathrm{~min}$, then $95^{\circ} \mathrm{C}$ for $10 \mathrm{~min}$. This was followed by a 2-step PCR program consisting of $95^{\circ} \mathrm{C}$ for $15 \mathrm{sec}$ and $60^{\circ} \mathrm{C}$ for $60 \mathrm{sec}$ for 35 cycles. Each sample was analyzed in triplicate, and SYBR Green fluorescence was detected using the Applied Biosystems 7900HT realtime PCR system. Data were analyzed with $2^{-\Delta \Delta C q}$ method (19). The experiment was repeated at least three times.

Cell culture. $\mathrm{CD}_{14}{ }^{+}$macrophages were prepared from tissues and peripheral blood mononuclear cells by antibody-coated microbeads (Miltenyi Biotec $\mathrm{GmbH}$ ), and the purity was routinely $>90 \%$ as assessed with PE-labeled anti-CD14 antibody (cat no., 557154; BD Biosciences, Franklin Lakes, NJ, USA) by flow cytometry using FlowJo software (version 7.5; FlowJo LLC, Ashland, OR, USA). Macrophages were cultured in vitro in RPMI-1640 medium (Invitrogen; Thermo Fisher 
Table II. Primer sequences for SYBR Green quantitative polymerase chain reaction.

\begin{tabular}{lll}
\hline Genes & \multicolumn{1}{c}{ Forward (5'-3') } & \multicolumn{1}{c}{ Reverse (5'-3') } \\
\hline Tbx21 & GGTTGCGGAGACATGCTGA & GTAGGCGTAGGCTCCAAGG \\
GATA-3 & GCCCCTCATTAAGCCCAAG & TTGTGGTGGTCTGACAGTTCG \\
RORC & GTGGGGACAAGTCGTCTGG & AGTGCTGGCATCGGTTCG \\
Foxp3 & GTGGCCCGGATGTGAGAAG & GGAGCCCTTGTCGGATGATG \\
BCL-6 & TGGTGACGCTTCAAAAGCCA & GCTAGAATAGACGATGTTCCCG \\
CXCR3 & CCACCTAGCTGTAGCAGACAC & AGGGCTCCTGCGTAGAAGTT \\
CXCL9 & TGCAATGAACCCCAGTAGTGA & GGTGGATAGTCCCTTGGTTGG \\
CXCL10 & TGAAATTATTCCTGCAAGCCAA & CAGACATCTCTTCTCACCCTTCTTT \\
GAPDH & GAAGGTGAAGGTCGGAGTC & GAAGATGGTGATGGGATTC \\
CD163 & AGTCCCATCTTTCACTCTGC & GCATCTTCTATGTCCCAGTG \\
IL-10 & GACTTTAAGGGTTACCTGGG & CTTGATGTCTGGGTCTTGGT \\
CD36 & TTGCAGGTCAATCTATGCTG & CTGGGTTTTCAACTGGAGAG \\
IL-12 $\beta$ & CACAACGGAATAGACCCAAA & TTAAATAGCATGAAGGCCCA \\
IL-1 3 & CCACCCTCTATCACTGACTT & CAAGGCTCAGTACATGCTCA \\
IL-6 & GATGCAATAACCACCCCTGA & TGACCAGAAGAAGGAATGCC \\
TNF- $\alpha$ & TGTACCTCATCTACTCCCAG & GAAGACCCCTCCCAGATAGA \\
$\beta$-actin & GCATCCACGAAACTACCTTC & GATCTCCTTCTGCATCCTGT
\end{tabular}

Tbx21, T-box 21; GATA3, GATA-binding protein 3; RORC, RAR-related orphan receptor c; Foxp3, Forkhead-box p3; BCL-6, B cell lymphoma 6 protein; CXCR3, CXC motif chemokine receptor 3; CXCL9, CXC motif chemokine ligand 9; CXCL10, CXC motif chemokine ligand 10; $\mathrm{CD}$, cluster of differentiation; IL, interleukin; TNF, tumor necrosis factor.

Scientific, Inc.) supplemented with $10 \%$ fetal calf serum (GE Healthcare Life Sciences) and granulocyte macrophage colony-stimulating factor (GM-CSF; $50 \mathrm{ng} / \mathrm{ml}$; R\&D Systems, Inc., Minneapolis, MN, USA). Following stimulation at 15, $30 \mathrm{~min}, 2,4$ and $24 \mathrm{~h}$, the cells were washed and stimulated with lipopolysaccharide (LPS; $100 \mathrm{ng} / \mathrm{ml}$; Sigma-Aldrich; Merck KGaA, Darmstadt, Germany) at $37^{\circ} \mathrm{C}$ for $16 \mathrm{~h}$, and the culture cells were collected for the analysis of interferon responsive factor (IRF)5 expression.

Western blot analysis. Cell pellets were lysed in ice-cold buffer containing a protease inhibitor cocktail (Roche Diagnostics, Basel, Switzerland). The lysates (10 mg/lane) were fractionated by $8-10 \%$ gradient SDS-PAGE. The lysates were subsequently transferred onto polyvinylidene difluoride membranes and blocked with $10 \%$ non-fat milk in PBS at room temperature for $1 \mathrm{~h}$ and analyzed by immunoblotting with specific antibodies at room temperature for $1 \mathrm{~h}$ to IRF5 (Cat\#13496; dilution 1:1,000; Cell Signaling Technology, Inc., Danvers, MA, USA) and $\beta$-actin (cat no., A1978; 1:2,000; Sigma-Aldrich; Merck KGaA). Subsequent to washing with $0.05 \%$ Tween-20 PBS, secondary horseradish peroxidase-conjugated antibodies (Cat\#31430; dilution 1:10,000; Pierce; Thermo Fisher Scientific, Inc.) were added and the blots were incubated at room temperature for $1 \mathrm{~h}$. The protein bands were visualized using enhanced chemiluminescence (Pierce; Thermo Fisher Scientific, Inc.).

Statistical analysis. Data are presented as the mean \pm standard error. Statistical analysis was performed using two-tailed Student t-test for unpaired data and two-way analysis of variance for multiple comparisons with a post hoc Fisher's Least Significant Difference test. SPSS (version 19; IBM Corp., Armonk, NY, USA) was used for statistical analysis. $\mathrm{P}<0.05$ was considered to indicate a statistically significant difference.

\section{Results}

Infiltration profile of immune cells in tumor and non-tumorous adjacent tissues of colorectal cancer. The profiles of infiltrating immune cells isolated from tumor and non-tumorous adjacent tissues obtained from patients with colorectal cancer was analyzed by qPCR amplification of each characteristic transcription factor of Th1, Th2, follicular T helper (Tfh), Treg and Th17 cells. It was revealed that significantly increased quantity of forkhead-box p3 (Foxp3) ${ }^{+}$Treg cells, Th1 cells and Tfh cells were present in tumor tissues compared with the adjacent tissues (Fig. 1A-H). No statistical difference in the number of Th2 (GATA3; Fig. 1D) and Th17 cells (RORC; Fig. 1B) was observed between tumor tissues and the adjacent tissues. This indicated that the profile of immune cells is distinct in the tumor tissues from the adjacent tissues. In addition, the expression of CXCR3, CXCL9 and CXCL10 were significantly increased in $\mathrm{T}$ cells isolated from tumor tissues compared with the adjacent tissues. This indicated that high expression of those molecules is associated with infiltration in colorectal cancer.

Distinctive patterns of infiltrating immune cells in tumor tissues with low and high infiltration. The infiltrating lymphocytes in tumor tissues from patients with colorectal cancer 

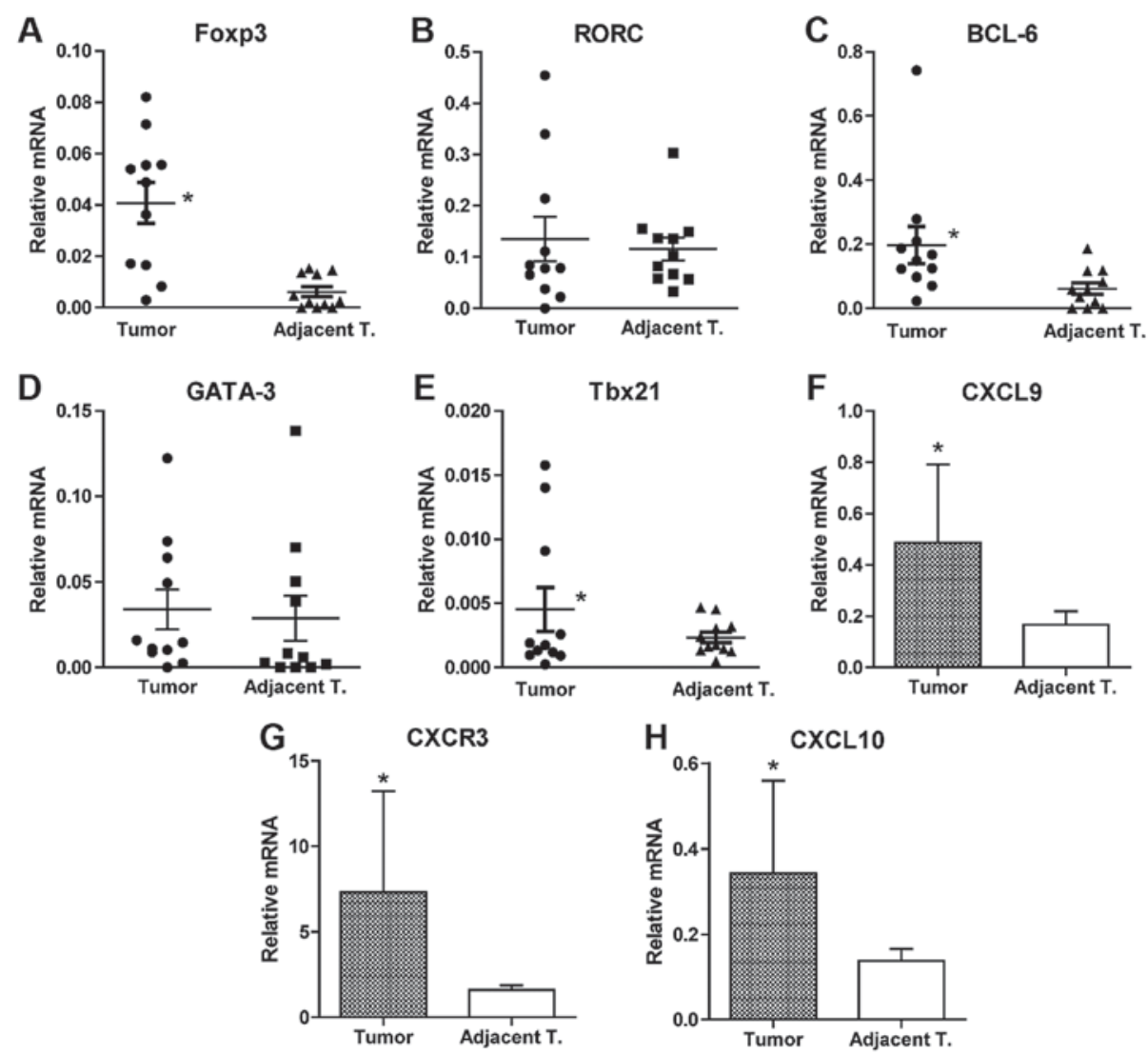

Figure 1. Analysis of the profile of infiltrating immune cells isolated from tumor and non-tumorous adjacent tissue. Immune cells were isolated from tissue blocks collected from selected patients with CRC during surgery via collagen IV digestion and gradient density centrifugation. Total RNA was extracted from the cells and subsequently reverse transcribed to cDNA. Specific primer sets were designed for transcription factors (A) Foxp3, (B) RORC, (C) BCL-6, (D) GATA-3 and (E) Tbx21 representing Treg, Th17, Tfh, Th2 and Th1 cells, respectively. qPCR was performed using the SYBR-Green method with specific primers to quantify the abundance of each subsets of infiltrating immune cells. GAPDH was amplified simultaneously for normalization. Data were analyzed using the $2^{-\triangle \Delta C q}$ method and presented as relative values to GAPDH. T cells were isolated from tumor and non-tumorous adjacent tissue of 6 selected patients with CRC using T cell-specific microbeads. qPCR was performed on RNA isolated from T cells for quantification of (F) CXCL9, (G) CXCR3 and (H) CXCL10. Data are presented as the mean \pm standard error of mean. Statistical analysis was performed using Student's t-test. "P $<0.05$, tumor tissue vs. non-tumorous adjacent tissue. qPCR, quantitative polymerase chain reaction, Tbx21, T-box 21; GATA3, GATA-binding protein 3; RORC, RAR-related orphan receptor c; Foxp3, Forkhead-box p3; BCL-6, B cell lymphoma 6 protein; CXCR3, CXC motif chemokine receptor 3; CXCL9, CXC motif chemokine ligand 9; CXCL10, CXC motif chemokine ligand 10; Treg, regulatory T cells; Th, T helper; Tfh, follicular T helper.

were counted. Furthermore, the expression of each specific transcription factor Foxp3, GATA-binding protein 3 (GATA3), T-box 21 (Tbx21) and RAR-related orphan receptor C (RORc) for each different $\mathrm{T}$ cell population, including Treg, Th2, Th1, Tfh and Th17 cells, were analyzed. As shown in Fig. 2, there were relatively a greater number of Treg cells and fewer Th1, Th17 and Tfh cells in tumor tissues with low infiltration $(<500$ cells/mg tissue) compared with tissues with medium (500-1,000 cells/mg tissue) and high infiltration (>1,000 cells/mg tissue). By contrast, in tissues with high infiltration $(>1,000$ cells $/ \mathrm{mg}$ tissue), there were an increased number of Th1, Th17 and Tfh cells and fewer Treg cells compared with tissues with low ( $<500$ cells/mg tissue) and medium ( $500-1,000$ cells/mg tissue) infiltration. The expression of CXCR3, CXCL9 and CXCL10 on $\mathrm{T}$ cells isolated from colorectal cancer tumor tissues was examined. As shown in Fig. 3, higher expression of CXCR3, CXCL9 and CXCL10 was observed on T cells isolated from tumor tissues with high infiltration compared with tumor tissues with low infiltration.

Characterization of tumor-infiltrating macrophages. To characterize the profile of macrophages in tumor tissue, the expression of major cytokines that are representative of M1 and M2 cells was analyzed by qPCR due to the limited number of isolated cells. The results revealed that tumor-infiltrating $\mathrm{CD}_{1}{ }^{+}$macrophages exhibited a dominant M2 phenotype as characterized by elevated expression of M2 marker genes, [interleukin (IL)-10, CD207, CD36 and CD163] compared with M1 marker genes [tumor necrosis factor (TNF) $\alpha$, IL-6, IL-1 $\beta$ and IL-12 $\beta$ (Fig. 4A).

Response of isolated $\mathrm{CD}_{14^{+}}$macrophages to GM-CSF stimulation. A total of three large tumor tissue blocks $(>50 \mathrm{mg})$ obtained from surgical patients with colorectal cancer were selected for isolation of $\mathrm{CD} 14^{+}$macrophages. Purified macrophages were stimulated with GM-CSF for different periods of time. It was demonstrated that macrophages from tumor tissues expressed markedly reduced IRF5, which is a characteristic transcription factor of M1 macrophages, compared with expression in peripheral macrophages isolated from peripheral blood of the same patient (Fig. 4B). Furthermore, tumor macrophages did not respond to the stimulation by GM-CSF, a driving cytokine for M1 macrophage differentiation, as measured by the expression of IRF5, whereas peripheral 


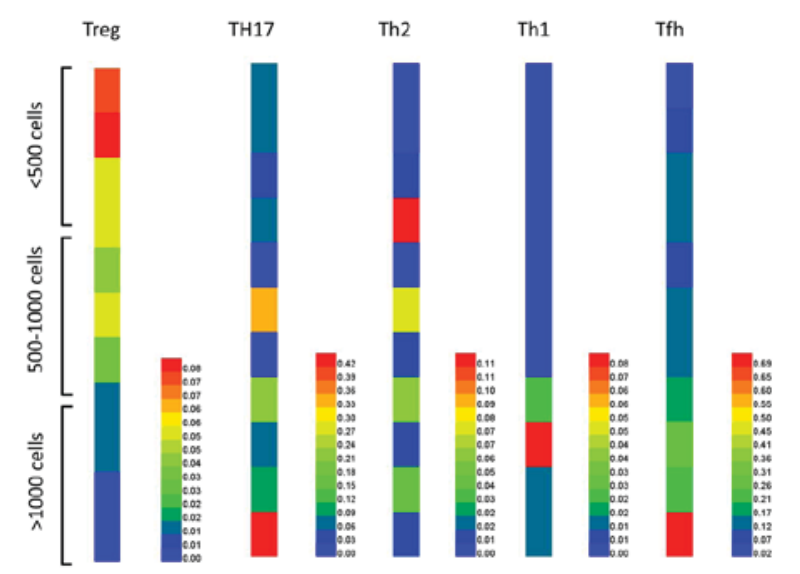

Figure 2. Association between the patterns of infiltrating immune cells with the intensity of infiltration in colorectal cancer tumor tissues. The numbers of infiltrating cells were enumerated using microscopy following isolation from tumor tissue with Percoll gradient centrifugation. The intensity of infiltration was set as low, medium and high with $<500,500-1,000$ and $>1,000$ infiltrating cells per $\mathrm{mg}$ of tissue, respectively. The cells were subject to quantitative polymerase chain reaction analysis for each characteristic transcription factors for each type of cell. The relative expression of mRNA was plotted using a heat map to illustrate the relevance of each type of cell to the intensity of infiltration. Red indicates high expression of mRNA of each specific subset of cells, while blue indicates low expression of mRNA. Th, T helper; Tfh, follicular $\mathrm{T}$ helper.

macrophages exhibited a strong response to GM-CSF stimulation after $24 \mathrm{~h}$ of stimulation (Fig. 4C). The results suggested that tumor macrophages were less inflammatory and refractory to conversion driven by M1 stimulating agents in colorectal cancer compared with peripheral macrophages.

\section{Discussion}

The most common treatment for colorectal cancer is surgery. In the case of localized tumors, surgery may completely eliminate the cancer. When the cancer has invaded the bowel wall or the lymph nodes, chemotherapy and/or immunotherapy are required to achieve the best benefits. Colorectal cancer is one of the major cancer types for which new immune-based cancer treatments are currently in development (20). The understandings of antitumor immune responses are crucial to the design and implement of immunomodulation for treatment.

The evaluation of immune infiltrates is even more complex due not only to the numerous cell types that can be found in tumors $(2,4)$, but also to the possibility that a given immune cell type can vary in terms of state of maturation and/or activation, and the fact that numerous diverse cell types can share similar markers (5). A CD4 T cell found in a tumor can be anergic, activated or regulatory. The same can be said for several other immune lineages (5). Tumor-infiltrating Foxp $3^{+}$regulatory $\mathrm{T}$ cells have also been shown to have a strong prognostic significance in colorectal cancer. Salama et al (21) reported that the density of regulatory $\mathrm{T}$ cells in normal and tumor tissue to be independent prognostic indicators, but not the density of $\mathrm{CD}^{+} \mathrm{T}$ cells. However, it has been reported elsewhere that Foxp $3^{+}$Treg cells were independent indicators of the prognosis of colorectal cancer (20). Di Giorgio et al (22) also revealed that the presence of lymphocytic infiltration in the tumor was associated with an improved prognosis by multivariate

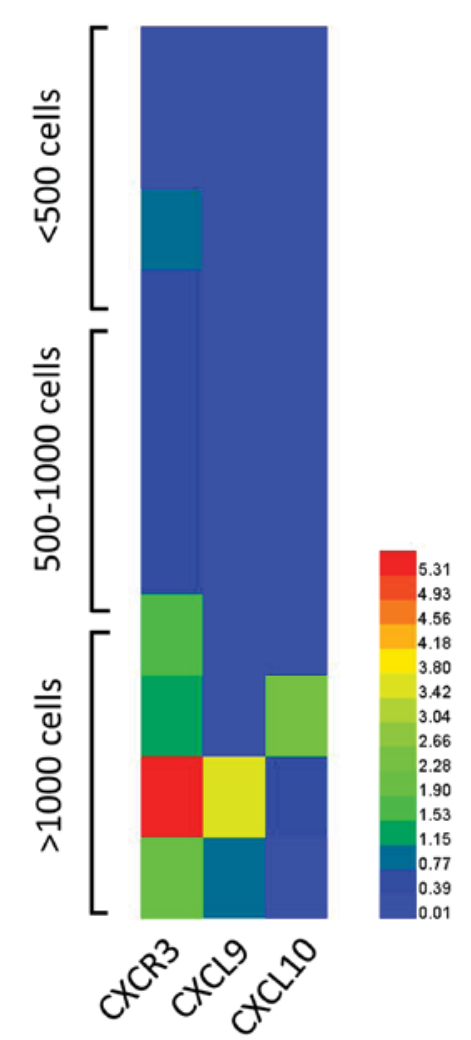

Figure 3. mRNA expression of CXCR3, CXCL9 and CXCL10 on T cells isolated from CRC tumor tissues. T cells were purified from post-digestion cell suspension of tumor tissue of colorectal cancer using anti-CD3 microbeads. The eluted cells were subject to lysis and purification of mRNA using a Qiagen RNA purification column. Quantitative polymerase chain reaction analysis was performed to determine the levels of RNA of each transcription factor, which represents each subset of immune cell. Data are presented as a heat map to associate the level of mRNA with the intensity of infiltration. CXCR3, CXC motif chemokine receptor 3; CXCL, CXC motif chemokine ligand.

analysis in patients with colorectal cancer resected between 1960 and 1978 ( $n=361 ; \mathrm{P}<0.001)$. A number of studies have also emphasized the location of immune infiltrate in tumors; CD8+ T cell infiltrates in cancer cell nests often were associated with improved prognosis when compared with those in cancer stroma and marginal regions $(1,4)$. Therefore, it will be more informative to describe a profile rather than emphasizing on a particular subset of immune cells in consideration of the complexity of immune infiltrates in colorectal cancer.

In the present study, the profiles of immune cells were analyzed, including Treg, Th1, Th2, Tfh and macrophages, and the profiles of low infiltration and high infiltration were compared. Profiles of tumor-infiltrating immune cells and immune cells in non-tumor adjacent tissues were also compared. However, these cells were also in different stages of differentiation, which was not addressed in the present study. The analysis of differentiation stages may provide further important information to define the profile of tumor-infiltrating immune cells. Notably, an increased number of Tfh cells were observed in the tumor tissue as compared with non-tumorous adjacent tissue, indicating significant involvement of B cell response in tumor tissues in colorectal cancer. The B cell response in tumor has been previously extensively studied in a number of types of cancer, including breast, ovarian and 

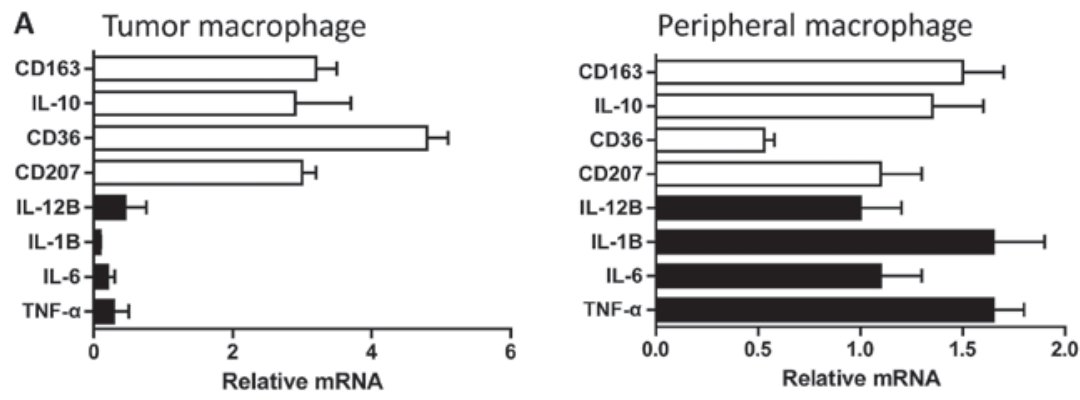

C

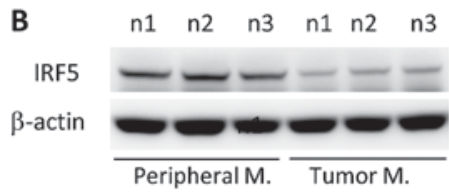

$\beta$-actin
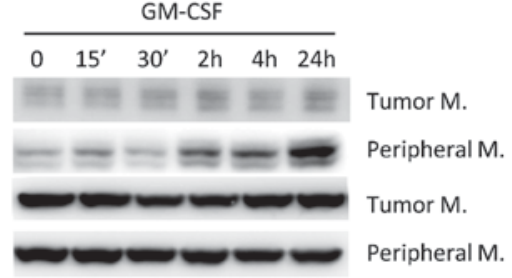

Figure 4. Phenotype of macrophages in tumor tissue of CRC and response of macrophages to GM-CSF stimulation in vitro. (A) Macrophages were purified from post-digestion cell suspension of tumor tissue and peripheral venous blood collected from patients with CRC using anti-CD14 microbeads. Total RNA was isolated from the purified cells and the mRNA levels of a panel of marker genes as indicated were determined by quantitative polymerase chain reaction. GAPDH was used for normalization. (B) A total of three specimens with sufficient number of infiltrating cells were selected, and the purified cells were lysed and subjected to the analysis of IRF5 at protein level with western blot analysis. (C) The purified macrophages were stimulated for different times as indicated in RPMI supplemented with $10 \%$ fetal calf serum and $50 \mathrm{ng} / \mathrm{ml}$ GM-CSF. Subsequently, the culture was stimulated with $100 \mathrm{ng} / \mathrm{ml}$ lipopolysaccharide at $16 \mathrm{~h}$ prior to western blot analysis of IRF5 expression. CD, cluster of differentiation; CRC, colorectal cancer; IL, interleukin; TNF $\alpha$, tumor necrosis factor $\alpha$; IRF5, interferon responsive factor 5; GM-CSF, granulocyte macrophage colony-stimulating factor.

non-small cell lung cancer (23). B cells exhibited evidence of somatic mutation and affinity maturation in breast cancer (23). In the present study, the increased number of Tfh cells indicated that local B cell differentiation occurred in tumor tissues. Consequently, it is likely the same scenario that occurred in colorectal cancer as that in breast cancer.

Macrophages are heterogeneous and comprise phenotypically and functionally distinct cell populations. With an increasing understanding of novel markers and differential roles of macrophages in the immune response, macrophages are characterized into different subsets. Different subsets require specific cytokine milieu for differentiation and maintenance and exhibit specific phenotypes and functions (24-30). Macrophage polarization is primarily determined by cytokines and ligands to pattern recognition receptors, including toll-like receptors (TLRs) on macrophages. Macrophages of the M1 phenotype are programmed to produce pro-inflammatory cytokines, including IL-12, IL-1 $\beta$, TNF $\alpha$ and IL-6, and perform a crucial role in the initiation and perpetuation of inflammatory response, whereas macrophages of the M2 phenotype exhibit anti-inflammatory properties characterized by the production of IL-10 and IL-13 and prominent phagocytosis $(26,28,30)$. Differentiation of M1 and M2 macrophages is driven by key cytokines, such as GM-CSF for M1 differentiation and M-CSF for M2 differentiation (28). By contrast, IFN- $\gamma$ or IL-4 primes initially differentiated macrophages and promotes their polarization (31). In addition, activation by LPS through TLR-4 augments the production of cytokines by macrophages (26). It was previously reported that IRF5 and IRF4 are the putative lineage determining transcription factors for M1 and M2 macrophages $(31,32)$. It has been shown that polarized M1 and M2 macrophages exhibit high plasticity and can be rendered to shift their phenotypes when the cytokine milieu changes. A balanced M1 to M2 ratio is required for the immune system homeostasis (27). In the present study, it was revealed that the macrophages of M2 phenotype isolated from tumor of colorectal cancer were refractory to in vitro converting to M1 phenotype, suggesting the defects of cells existed or the anergic state of cells. Current research to develop emerging immunotherapies that target the dysregulated M1/M2 macrophages is considered to make significant advances in cancer immunotherapy. Understanding the preferential accumulation of macrophages in a specific type of cancer would greatly support the future application of macrophage-directed immunotherapy. Although current agents such as Coley's toxins that stimulate the growth of M1 macrophages involve great side effects $(33,34)$, new mediators that stimulate and maintain M1 macrophages will begin a new chapter in cancer therapy, and in such cases colorectal cancer may be a good candidate for macrophage-directed immunotherapy.

CXCR3, CXCL9 and CXCL10 were associated with the intensity of infiltration of $\mathrm{T}$ cells to tumor microenvironment. Zeste homologue 2 (EZH2)-mediated suppression of Th1-type chemokines CXCL9 and CXCL10 determine effector T cell trafficking to the tumor microenvironment (35). Treatment with epigenetic modulators such as EZH2 inhibitor removes the repression and increases effector $\mathrm{T}$ cell tumor infiltration, slows down tumor progression, and improves the therapeutic efficacy of programmed death-ligand 1 (PD-L1; also termed B7-H1) checkpoint blockade and adoptive $\mathrm{T}$ cell transfusion in tumor-bearing mice $(35,36)$. In colorectal cancer, it was demonstrated that high expression of CXCR3, CXCL9 and CXCL10 on T cells was associated with high infiltration ( $>1,000$ cells $/ \mathrm{mg})$. By analyzing the expression of these chemokines, the present results suggested the clinical specimens can be categorized into different groups that may be sensitive or insensitive to PD-L1 immunotherapy.

In the present study, it was identified that Th1 and Tfh cells, as well as M2 macrophages, are dominant cells in colorectal cancer tumors. The results of the present study suggest that the 
analysis of the profile of intratumor immune cells may assist the prediction of prognosis.

\section{Acknowledgements}

The present study was supported by the Changzhou Municipal Scientific Research grant (grant no. CE20125020).

\section{References}

1. Chiba T, Ohtani H, Mizoi T, Naito Y, Sato E, Nagura H, Ohuchi A, Ohuchi K, Shiiba K, Kurokawa Y and Satomi S: Intraepithelial CD8+ T-cell-count becomes a prognostic factor after a longer follow-up period in human colorectal carcinoma: Possible association with suppression of micrometastasis. Br J Cancer 91: 1711-1717, 2004.

2. Diederichsen AC, Hjelmborg Jv, Christensen PB, Zeuthen J and Fenger C: Prognostic value of the CD4+/CD8+ ratio of tumour infiltrating lymphocytes in colorectal cancer and HLA-DR expression on tumour cells. Cancer Immunol Immunother 52: 423-428, 2003.

3. Funada Y, Noguchi T, Kikuchi R, Takeno S, Uchida Y and Gabbert HE: Prognostic significance of CD8+ T cell and macrophage peritumoral infiltration in colorectal cancer. Oncol Rep 10: 309-313, 2003.

4. Naito Y, Saito K, Shiiba K, Ohuchi A, Saigenji K, Nagura H and Ohtani H: CD8+ T cells infiltrated within cancer cell nests as a prognostic factor in human colorectal cancer. Cancer Res 58: 3491-3494, 1998.

5. Oberg A, Samii S, Stenling R and Lindmark G: Different occurrence of $\mathrm{CD} 8+, \mathrm{CD} 45 \mathrm{R} 0+$, and $\mathrm{CD} 68+$ immune cells in regional lymph node metastases from colorectal cancer as potential prognostic predictors. Int J Colorectal Dis 17: 25-29, 2002.

6. Ropponen KM, Eskelinen MJ, Lipponen PK, Alhava E and Kosma VM: Prognostic value of tumour-infiltrating lymphocytes (TILs) in colorectal cancer. J Pathol 182: 318-324, 1997.

7. Nagorsen D, Keilholz U, Rivoltini L, Schmittel A, Letsch A, Asemissen AM, Berger G, Buhr HJ, Thiel E and Scheibenbogen C: Natural T-cell response against MHC class I epitopes of epithelial cell adhesion molecule, her-2/neu, and carcinoembryonic antigen in patients with colorectal cancer. Cancer Res 60: 4850-4854, 2000.

8. Nagorsen D, Scheibenbogen C, Marincola FM, Letsch A and Keilholz U: Natural T cell immunity against cancer. Clin Cancer Res 9: 4296-4303, 2003.

9. Nagorsen D, Scheibenbogen C, Letsch A, Germer CT, Buhr HJ, Hegewisch-Becker S, Rivoltini L, Thiel E and Keilholz U: T cell responses against tumor associated antigens and prognosis in colorectal cancer patients. J Transl Med 3: 3, 2005.

10. Blankenstein T: The role of tumor stroma in the interaction between tumor and immune system. Curr Opin Immunol 17: 180-186, 2005.

11. Kinnula VL, Torkkeli T, Kristo P, Sormunen R, Soini Y, Pääkkö P, Ollikainen T, Kahlos K, Hirvonen A and Knuutila S: Ultrastructural and chromosomal studies on manganese superoxide dismutase in malignant mesothelioma. Am J Respir Cell Mol Biol 31: 147-153, 2004.

12. Kojima M, Morisaki T, Tsukahara Y, Uchiyama A, Matsunari Y, Mibu R and Tanaka M: Nitric oxide synthase expression and nitric oxide production in human colon carcinoma tissue. J Surg Oncol 70: 222-229, 1999.

13. Nakamura $\mathrm{Y}$, Yasuoka $\mathrm{H}$, Tsujimoto $\mathrm{M}$, Yoshidome $\mathrm{K}$, Nakahara M, Nakao K, Nakamura M and Kakudo K: Nitric oxide in breast cancer: Induction of vascular endothelial growth factor- $\mathrm{C}$ and correlation with metastasis and poor prognosis. Clin Cancer Res 12: 1201-1207, 2006.

14. Ahmed B and Van Den Oord JJ: Expression of the inducible isoform of nitric oxide synthase in pigment cell lesions of the skin. Br J Dermatol 142: 432-440, 2000.

15. Masri FA, Comhair SA, Koeck T, Xu W, Janocha A, Ghosh S, Dweik RA, Golish J, Kinter M, Stuehr DJ, et al: Abnormalities in nitric oxide and its derivatives in lung cancer. Am J Respir Crit Care Med 172: 597-605, 2005.

16. Vakkala M, Kahlos K, Lakari E, Pääkkö P, Kinnula V and Soini Y: Inducible nitric oxide synthase expression, apoptosis, and angiogenesis in in situ and invasive breast carcinomas. Clin Cancer Res 6: 2408-2416, 2000.
17. Kono K, Salazar-Onfray F, Petersson M, Hansson J, Masucci G, Wasserman K, Nakazawa T, Anderson P and Kiessling R: Hydrogen peroxide secreted by tumor-derived macrophages down-modulates signal-transducing zeta molecules and inhibits tumor-specific T cell-and natural killer cell-mediated cytotoxicity. Eur J Immunol 26: 1308-1313, 1996.

18. Rotondo R, Mastracci L, Piazza T, Barisione G, Fabbi M, Cassanello M, Costa R, Morandi B, Astigiano S, Cesario A, et al: Arginase 2 is expressed by human lung cancer, but it neither induces immune suppression, nor affects disease progression. Int J Cancer 123: 1108-1116, 2008.

19. Livak KJ and Schmittgen TD: Analysis of relative gene expression data using real-time quantitative PCR and the 2(-Delta Delta C(T)) method. Methods 25: 402-408, 2001.

20. Jochems C and Schlom J: Tumor-infiltrating immune cells and prognosis: The potential link between conventional cancer therapy and immunity. Exp Biol Med (Maywood) 236: 567-579, 2011.

21. Salama P, Phillips M, Grieu F, Morris M, Zeps N, Joseph D, Platell C and Iacopetta B: Tumor-infiltrating FOXP3+ T regulatory cells show strong prognostic significance in colorectal cancer. J Clin Oncol 27: 186-192, 2009.

22. Di Giorgio A, Botti C, Tocchi A, Mingazzini P and Flammia M: The influence of tumor lymphocytic infiltration on long term survival of surgically treated colorectal cancer patients. Int Surg 77: 256-260, 1992.

23. Nzula S, Going JJ and Stott DI: Antigen-driven clonal proliferation, somatic hypermutation, and selection of B lymphocytes infiltrating human ductal breast carcinomas. Cancer Res 63: 3275-3280, 2003

24. Biswas SK and Mantovani A: Macrophage plasticity and interaction with lymphocyte subsets: Cancer as a paradigm. Nat Immunol 11: 889-896, 2010.

25. Fleetwood AJ, Lawrence T, Hamilton JA and Cook AD: Granulocyte-macrophage colony-stimulating factor (CSF) and macrophage CSF-dependent macrophage phenotypes display differences in cytokine profiles and transcription factor activities: Implications for CSF blockade in inflammation. J Immunol 178: 5245-5252, 2007.

26. Gordon S and Martinez FO: Alternative activation of macrophages: Mechanism and functions. Immunity 32: 593-604, 2010.

27. Mantovani A, Sica A and Locati M: Macrophage polarization comes of age. Immunity 23: 344-346, 2005.

28. Martinez FO, Gordon S, Locati $M$ and Mantovani A: Transcriptional profiling of the human monocyte-to-macrophage differentiation and polarization: New molecules and patterns of gene expression. J Immunol 177: 7303-7311, 2006.

29. Martinez FO, Helming L and Gordon S: Alternative activation of macrophages: An immunologic functional perspective. Annu Rev Immunol 27: 451-483, 2009.

30. Stein M, Keshav S, Harris N and Gordon S: Interleukin 4 potently enhances murine macrophage mannose receptor activity: A marker of alternative immunologic macrophage activation. J Exp Med 176: 287-292, 1992.

31. Krausgruber T, Blazek K, Smallie T, Alzabin S, Lockstone H, Sahgal N, Hussell T, Feldmann M and Udalova IA: IRF5 promotes inflammatory macrophage polarization and TH1-TH17 responses. Nat Immunol 12: 231-238, 2011.

32. Satoh T, Takeuchi O, Vandenbon A, Yasuda K, Tanaka Y, Kumagai Y, Miyake T, Matsushita K, Okazaki T, Saitoh T, et al: The Jmjd3-Irf4 axis regulates M2 macrophage polarization and host responses against helminth infection. Nat Immunol 11: 936-944, 2010.

33. Brunschwig A: The Efficacy of 'Coley's Toxin' in the Treatment of Sarcoma: An experimental study. Ann Surg 109: 109-113, 1939.

34. Maletzki C, Klier U, Obst W, Kreikemeyer B and Linnebacher M: Reevaluating the concept of treating experimental tumors with a mixed bacterial vaccine: Coley's toxin. Clin Dev Immunol 2012: $230625,2012$.

35. Zhao E, Maj T, Kryczek I, Li W, Wu K, Zhao L, Wei S, Crespo J, Wan S, Vatan L, et al: Cancer mediates effector T cell dysfunction by targeting microRNAs and EZH2 via glycolysis restriction. Nat Immunol 17: 95-103, 2016.

36. Peng D, Kryczek I, Nagarsheth N, Zhao L, Wei S, Wang W, Sun Y, Zhao E, Vatan L, Szeliga W, et al: Epigenetic silencing of TH1-type chemokines shapes tumour immunity and immunotherapy. Nature 527: 249-253, 2015. 